\title{
International Normalized Ratio of Prothrombin Time
}

National Cancer Institute

\section{Source}

National Cancer Institute. International Normalized Ratio of Prothrombin Time. NCI

Thesaurus. Code C64805.

A measure of the extrinsic pathway of coagulation. The International Normalized Ratio of Prothrombin Time (INR) is the ratio of a patient prothrombin time to a normal (control) sample raised to the power of the International Sensitivity Index (ISI) with a range of 0.8 to 1.2 seconds. 